\title{
Video Big Data Analytics in the Cloud: Research Issues and Challenges *
}

\author{
Aftab Alam ${ }^{1}$, Shah Khalid ${ }^{2}$, Muhammad Numan Khan ${ }^{1}$, Tariq Habib Afridi ${ }^{1}$, \\ Irfan Ullah ${ }^{1}$, and Young-Koo Lee ${ }^{1}$ \\ 1 Department of Computer Science and Engineering, Kyung Hee University (Global \\ Campus), Yongin 1732, South Korea \\ \{aftab, numan, afridi,irfan, yklee\}@khu.ac.kr \\ 2 School of Computer Science and Communication Engineering, Jiangsu University
}

\{shahkhalid\}@ujs.edu.cn

\begin{abstract}
On the rise of distributed computing technologies, video big data analytics in the cloud have attracted researchers and practitioners' attention. The current technology and market trends demand an efficient framework for video big data analytics. However, the current work is too limited to provide an architecture on video big data analytics in the cloud, including managing and analyzing video big data, the challenges, and opportunities. This study proposes a service-oriented layered reference architecture for intelligent video big data analytics in the cloud. Finally, we identify and articulate several open research issues and challenges, which have been raised by the deployment of big data technologies in the cloud for video big data analytics. This paper provides the research studies and technologies advancing the video analyses in the era of big data and cloud computing. This is the first study that presents the generalized view of the video big data analytics in the cloud to the best of our knowledge.
\end{abstract}

Keywords: big data - intelligent video analytics · cloud-based video analytics system · video analytics review · deep learning · distributed computing · intermediate results orchestration · cloud computing.

\section{Introduction}

Videos are recorded and uploaded to the cloud on a regular base. Many sources include CCTV, smartphones, drones, satellites, etc. They are actively contributing to video generation, leading to the evolution of video analytics and management systems. Video management and services providers such as Facebook [14, and YouTube [39], are considered as valuable sources of large-scale video data. Along with these, various leading industrial organizations have successfully deployed video management and analytics platforms that provide more bandwidth

\footnotetext{
^ This work was supported by the Institute for Information and Communications Technology Promotion Grant through the Korea Government (MSIT) under Grant R7120-17-1007 (SIAT CCTV Cloud Platform).
} 
and high-resolution cameras collecting videos at scale and has become one of the latest trends in the video surveillance industry. For example, more than 400 hours of videos are uploaded in a minute on Youtube 32, and more than one hundred and seventy million video surveillance cameras have been installed in china only [30. It has been reported that the data generated by various Internet of Things (IoT) devices will see a growth rate of $28.7 \%$ over the period $2018-2025$, where surveillance videos are the majority shareholder 8 .

Such an enormous video data is considered "big data" because various sources generate a large volume of video data at high velocity that holds high Value. Even though $65 \%$ of the big data shares hold by surveillance videos are monitored, but still, a significant portion of video data has been failed to notice 19. That neglected data contain valuable information directly related to real-world situations. Video data provide information about interactions, behaviors, and patterns, whether its traffic or human patterns. However, handling such a large amount of complex video data is not worthwhile utilizing traditional data analytical approaches. Therefore, more comprehensive and sophisticated solutions are required to manage and analyses such unstructured video big data.

Due to the data-intensive and resources hungry nature of large-scale video data processing, extracting the video's insights is a challenging task. A considerable video data video poses significant challenges for video management and mining systems that require powerful machines to deal with video big data. Moreover, a flexible solution is necessary to store and mine this large volume of video data for decision making. However, large-scale video analytics becomes a reality due to the popularity of big data and cloud computing technologies.

Cloud computing is an infrastructure for providing convenient and ubiquitous remote access to a shared pool of configurable computing resources. These resources can be managed with minimal management effort or service. Big data technologies, such as Hadoop or Spark echo system, are software platforms designed for distributed computing to process, analyze, and extract the valuable insights from large datasets in a scalable and reliable way. The cloud is preferably appropriate to offer the big data computation power required to process these large datasets. Amazon web service [4] and Oracle Big Data Analytics 13 are some examples of big data analytics platforms. When video analytics solutions are provided in a cloud computing environment, then it is called Cloud-based Video Analytics System (CVAS). Large-scale video analytics in the cloud is a multi-disciplinary area, and the next big thing in big data, which opens new research avenues for researchers and practitioners.

This work aims to conduct a comprehensive study on the status of largescale video analytics in the cloud-computing environment while deploying video analytics techniques. First, a lambda style [27] service-oriented reference architecture called Lambda CVAS (L-CVAS) has been proposed for video big data analytics in the cloud. Then open research issues and challenges are discussed, with a focus on proposed architecture. 


\section{Proposed L-CVAS Architecture}

Fig. 1 presents the proposed L-CVAS reference architecture for distributed Intelligent Video Analytics (IVA) in the cloud. It is composed of five layers i.e., Video Big Data Curation Layer (VBDCL), Video Big Data Processing Layer (VBDPL), Video Big Data Mining Layer (VBDML), Knowledge Curation Layer (KCL), and Web Service Layer (WSL).

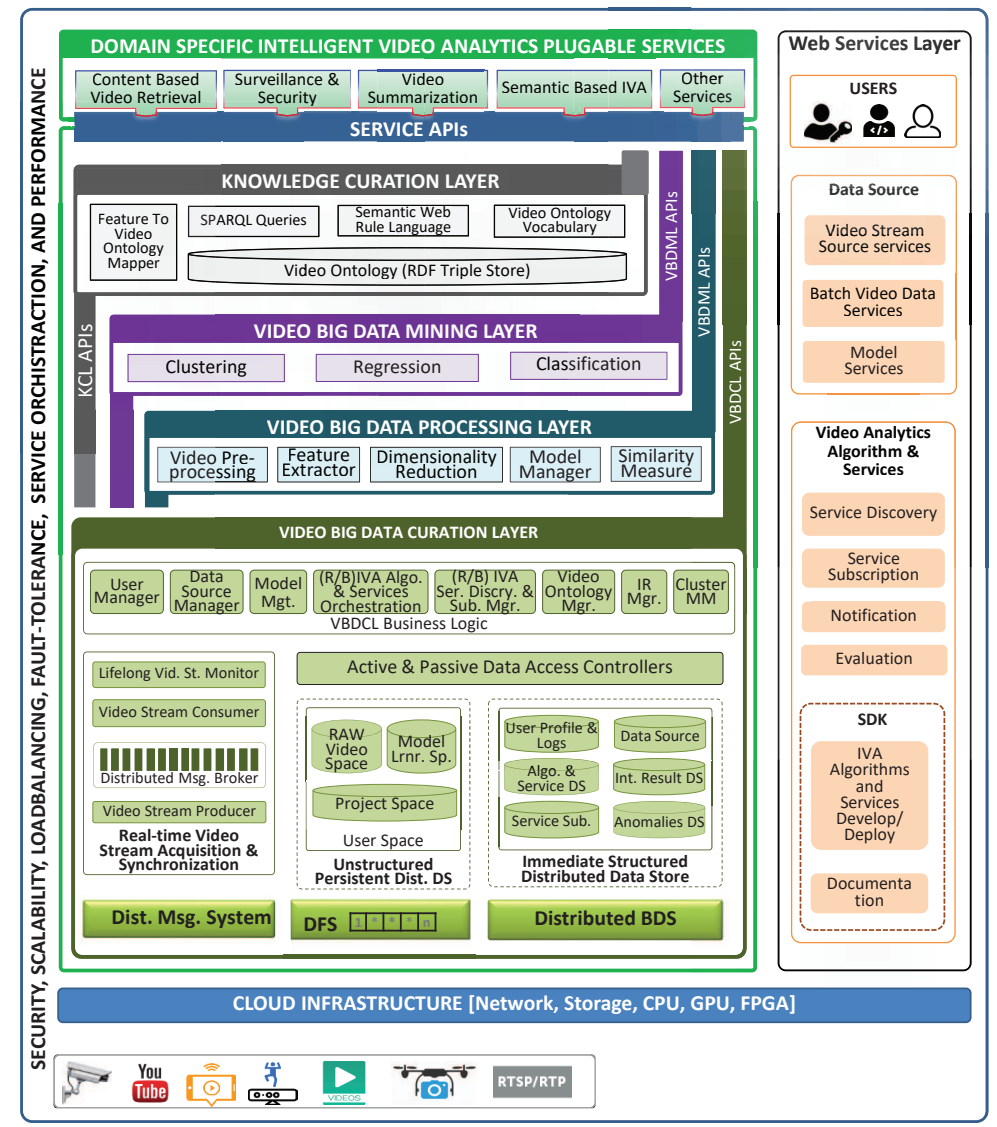

Fig. 1. A reference architecture for intelligent video analytics in the cloud.

\subsection{Video Big Data Curation Layer}

Effective data management is key to extract insights from the data. It is a petascale storage architecture that can be accessed in a highly efficient and convenient manner. We design the VBDCL to manage video big data efficiently. VBDCL's 
consists of three main components: Real-time Video Stream Acquisition and Synchronization (RVSAS), Distributed Persistent Data Store (DPDS), and VBDCL Business Logic.

Real-time Video Stream Acquisition and Synchronization To handle large-scale video stream acquisition in real-time, to manage the Intermediate Results (IR), anomalies, and the communication among Real-time IVA (RIVA) services, we design the RVSAS component while assuming a distributed messaging system. RVSAS provides client APIs on the top of a distributed messaging system for the proposed framework. Distributed Message Broker, e.g., Apache Kafka 23], is an independent application responsible for buffering, queuing, routing, and delivering the messages to the consumers being received from the message producer. The RVSAS component is responsible for handling and collecting real-time video streams from device-independent video data sources. Once the video stream is acquired, then it is sent temporarily to the distributed broker server. The worker system, on which an IVA service is configured, e.g., activity recognition, reads the data from the distributed broker and process. The RVSAS component is composed of five sub-modules, i.e., Distributed Message Broker Manager (DMBM), Video Stream Producer (VSP), Video Stream Consumer (VSC), Intermediate Results Manager (IRM), and Lifelong Video Stream Monitor (LVSM).

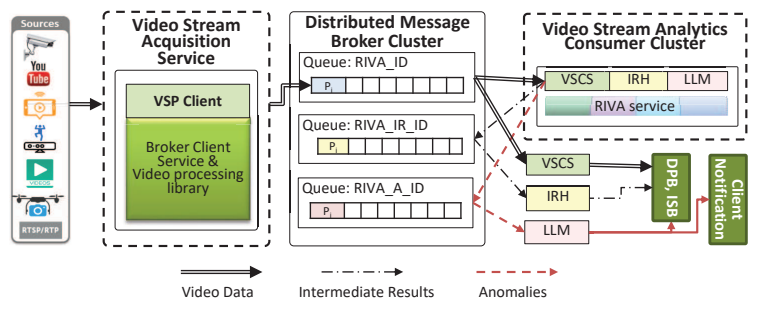

Fig. 2. Real-time Video Stream Acquisition and Synchronization

DMBM are used to manage the queues in the distributed message broker cluster considering RIVA services. Three types of queues, RIVA_ID, RIVA_IR_ID, and RIVA_A_ID as shown in Fig. 2, are automatically generated by the DMBM module on the distributed message broker when a new real-time RIVA service is created. Here RIVA, ID, IR, and A stands for RIVA service, unique identifier of the service, IR, and Anomalies, respectively. These queues are used to hold the actual video stream being acquired by VSP, IR produced by an algorithm, and anomalies detected by the video analytics services. The VSP module is used to provide interfaces to video stream data sources, acquire large-scale streams from device-independent video data sources, and send it to the broker server queue, i.e., RIVA_ID, in the form of compressed mini-batches. The VSC assists the RIVA service to read the mini-batches of the video stream from the respective queue for 
analytics, as shown in Fig. 2. A RIVA is subject to produces two types of results, i.e., intermediate results and anomalies. In this context, RIVA utilizes VSC and LVSM to sent the intermediate results and anomalies to the RIVA_IR_ID, and RIVA_A_ID queues respectively.

Immediate Structured Distributed Datastore The Immediate Structured Distributed Data Store (ISDDS) is provided to manage large-scale structured data in the distributed environment over Distributed Big Datastore (DBDS). Because of the data-intensive operation and according to the other layer's requirements, technologically, a distributed big data store can be deployed such as Cassandra, HBase [37, etc. The ISDDS hosts five types of data. Role-based user logs are maintained through the User Profile and Logs meta-store. The proposed framework manages two types of video data sources through the Data Source meta-store. These are video data sources, such as IP-cameras, Kinect, body-worn cameras, etc., and batch video datasets. The former can be subscribed to RIVA service while the latter is eligible for Batch IVA (BIVA) services. The metainformation of these sources, along with access rights, are managed through the Data Source meta-store. Administrator and developer roles can develop, create, and deploy video analytics algorithms through the L-CVAS. Similarly, different IVA algorithms can be pipelined into an IVA service. The management of video analytics algorithms and services is managed through Video Analytics Algorithm and Service meta-store, respectively. As stated that in IVA pipelining environment, the output of one IVA algorithm can be the output of another algorithm. In this context, we design a general container called IR datastore to persist and index the output of an IVA algorithm and services. Finally, the users are allowed to subscribe to the data sources to the IVA services. The subscription information is maintained through the Subscription meta-store, and the anomalies are maintained through the Anomalies meta-store.

Unstructured Persistent Distributed Datastore The Unstructured Persistent Distributed Data Store (UPDDS) component built on the top of the Distributed File System (DFS), such as Hadoop File System (HDFS), that facilitates permanent and distributed big-data storage. Upon new registration, a formal User Space is created on the top of DFS. The User Space is managed through a proper hierarchical directory structure, and special read and writes access are granted to the owner. All the directories are synchronized and mapped in the corresponding user profile logs. Under the User Space, three types of distributed directories are created, i.e., Raw Video Space, Model Space, and Project Space. Raw Video Space is used for the management of the video data. The Model Space is provided to facilitate the developers to manage the training and testing model according to the deployed IVA algorithm. The Project Space is provided to manage the source code of the respective developer and practitioners.

Active and Passive Data Readers and Writer This module gives readwrite access to the underlying data securely according to the business logic of 
the VBDCL Business Logic and according to the registered user access rights. This sub-module is composed of two types of readers and writers, i.e., Active and Passive Data Reader, which are used to access ISDDS and UPDDS.

ISBDS Business Logic The actual business logic is provided by the VBDCL Business Logic, which implements seven modules. The User Manager module encapsulates all the user-related operations such as new user account creation, access role assignment, and session management. Through the Data Source Manager and Model Manager modules, the user can manage the Video Stream Data Source (VSDS), video data uploading, and model management. The (R/B) IVA Algorithm and Service Manager are built to manage, develop, and deploy new IVA algorithms and services, respectively. The former is provided as-a-Service (aaS) to the developers, while the latter is provided aaS to the consumers. The developer role can create and publish a new video analytics algorithm. The algorithm is then made available aaS to other developers and can use it. Once IVA services are created, then the users are allowed to subscribe to the streaming video data sources and batch data against the provided RIVA and BIVA services, respectively, using the Service Discovery and Subscription Manager. The IR Manager provides a secure way of getting the IR and maps it according to ontology. Finally, to provide the proposed system's functionality over the web, it incorporates top-notch functionality into simple unified role-based web services. The Web Service Layer is built on the top of VBDCL Business Logic.

\subsection{Video Big Data Processing Layer}

IVA requires video data pruning and strong feature extraction. With such intentions, the VBDPL layer consists of three components, i.e., Video Pre-processing, Feature Extractor, and Dimensionality Reduction.

Video Pre-processing component is designed to clean and remove noise from videos. It is supposed to deploy several distributed video pre-processing operations, including frame extraction, frame-resizing, frame-conversion from RGB to grayscale, shot boundary detection, segmentation, transcoding, etc. In the first step, frames are extracted from a video for processing. The number of frames to be extracted is dependent on the user objective and task-dependent. Candidate frames can be all frames, step frames (every second frame, fifth frame, etc.), or keyframes. The spatial operations highly depend on the scenario and objective. Spatial operations include frame resizing (for reducing computational complexity), corrections (brightness, contrast, histogram equalization, cropping, keyframes), mode (RGB, Grayscale, etc.), and many other operations. Segmentation is used for various purposes, such as partitioning video into semantically related chunks.

The Feature Extractor extracts the features from the raw videos that can be interpreted by the Machine Learning (ML) algorithm. In this context, several feature extraction algorithms have been introduced for video data. These feature extraction approaches can be categorized into static features of keyframes, 
object features, dynamic/motion feature extraction, trajectory-based features extraction, and deep learning-based feature extraction [36].

The Feature selection and dimensionality reduction reduce the size of the features. Large sizes of feature sets are expensive in terms of time for training and/or performing classification acquired by trained classifiers. For example, Principal Component Analysis (PCA) and its variants are used to reduce the size of features. During feature selection, most relevant features are selected by discarding irrelevant and weak features. Inappropriate or partially relevant features can negatively affect model performance. Therefore, only a limited set of features should be selected and used for training classifiers. This is what precisely the purpose of this component is and deploy different algorithms in this context. Similarly, some feature reduction techniques available that selects the specific set of limited features in real-time.

\subsection{Video Big Data Mining Layer}

The VBDML utilizes diverse types of machine-learning algorithms, i.e., supervised, semi-supervised, and unsupervised algorithms to find different type of information from the videos 38. In this context, VBDML layer hosts three types of components, i.e., Classification, Regression, Clustering.

The classification component provides various ML algorithms, e.g. Support Vector Machine (SVM), Nearest Neighbors, Random Forest, Decision Tree, Naïve Bayes, etc., that identifies that a particular object in a video frame belongs to which category while using predefined classes. The Regression component includes algorithms, e.g., Linear Regression, Decision Tree Regression, Logistic Regression, and many more., which predict a continuous-valued attributed associated with objects rather than discrete values. The Clustering component encapsulates algorithms, e.g., K-Mean, spectral clustering, etc. that produces data groups depending upon the similarity of data items.

Distributed Deep Learning for IVA Recently, Convolutional Neural Network $(\mathrm{CNN})$ based approaches have shown performance superiority in tasks like optical character recognition [5], and object detection [26]. The motive of the deep learning is to scale the training in three dimensions, i.e., size and complexity of the models [10], proportionality of the accuracy to the amount of training data [18, and the hardware infrastructure scalability [44. A CNN or ConvNet is a type of neural network that can recognize visual patterns directly from the pixels of images with less preprocessing. CNN based video classification methods have been proposed in the literature to learn features from raw pixels from both short video and still images [0]. In the proposed L-CVAS framework, both the VBDPL, and VBDML are capable to deploy deep-learning approaches for distributed IVA. Since on the dawn of deep learning, various open-source architecture have been developed. Some of the well-known and state-of-the-art CNN architectures are AlexNet [24, GoogleNet [34, VGGNet 33, and ResNet [17.

There are two approaches for leveraging DL in distributed computing, i.e., model and data distribution [28. In the former, the DL model is partitioned in 
logical fragments and loaded to different worker agents for training, as shown in Fig. 3 3 . The training data are input to the work-agent(s) that have the input layer. In the second approach, the deep learning model is replicated to all the cluster's worker-agents, as shown in Fig. 3b. The training dataset is partitioned into non-overlapping sub-dataset, and each sub-dataset are loaded to the different worker-agents of the cluster. Each worker-agent executes the training on its sub-dataset of training data. The model parameters are synchronized among the cluster worker-agents to updates the model parameters. The data distribution approached naturally fits in the distribute computing MapReduce paradigm [12].

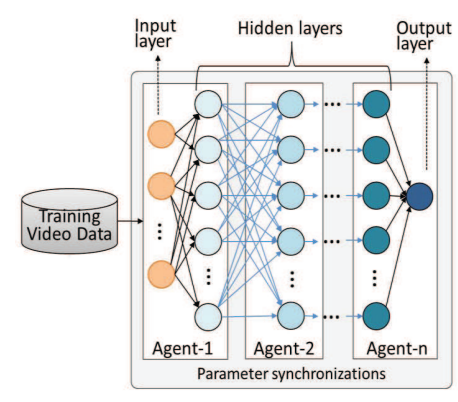

(a)

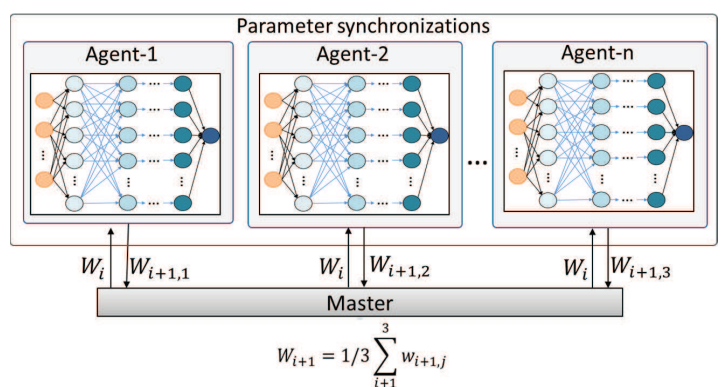

(b)

Fig. 3. (a) Scalable deep learning utilizing model distribution. (b) Scalable deep learning utilizing data distribution.

Big Data Engines, ML Libraries, and IVA The VBDPL, and VBDML are assumed to be built on the top of distributed computing engines. Hadoop MapReduce [11 is a distributed programming model developed for data-intensive tasks. Apache Spark follows a similar programming model like MapReduce but extends it with Resilient Distributed Datasets (RDDs), data sharing abstraction 42]. Hadoop's MapReduce operations are heavily dependent on the hard disk while Spark is based on in-memory computation, making Spark a hundred times faster than Hadoop [42. Spark support interactive operations, Directed Acyclic Graph (DAG) processing, and process streaming data in the form of mini-batches in near real-time 41. Apache Spark is batch centric and treats stream processing as a special case, lacking support for cyclic operations, memory management, and windows operators. Such issues of Spark has been elegantly addressed by Apache Flink [6. Apache Flink treats batch processing as a special and does not use micro-batching. Similarly, Apache Storm and Samza is another prominent solution focused on working with large data flow in real-time.

To achieve scalability, big data techniques can be exploited by existing video analytics modules. The VBDPL is not provided by default and needs its implementation on the top of these big data engines. However, The ML approaches 
can be categorized into two classes in the context of VBDML. One class reimplements the existing ML task by providing a middleware layer to run them on a big data platform. This general type of middleware layer provides general primitives/operations that assists in various learning tasks. Users who want to try different ML algorithms in the same framework can take benefits from it. In the second class, the individual video analytics and ML algorithm are executed on a big data platform that is directly built on top of a big data engine for better scalability. Spark MLlib [29, Mahout [31, FlinKML 6] are list of some open-source ML packages built on the top of Hadoop, Apache Spark and Flink, respectively, that support many scalable learning algorithms. For deep learning, various open-source libraries have been develop including TensorFlow [1, DeepLearning4J [35, Keras [7, BigDL [9], and PyTorch [20].

\subsection{Knowledge Curation Layer}

The KCL layer has been proposed under CVAS architecture, on the top of VBDML, which map the IR (both online and offline) into the video ontology in order to allow domain-specific semantic video and complex event analysis. The KCL is composed of five components, i.e., Video Ontology Vocabulary, Video Ontology, Semantic Web Rules, FeatureOnto Mapper, and SPARQL queries. Video Ontology Vocabulary standardizes the basic terminology that governs the video ontology, such as concept, attributes, objects, relations, video temporal relation, video spatial relation, and events. Video Ontology is a generic semantic-based model for the representation and organization of video resources that allow the CVAS users for contextual complex, event analysis, reasoning, search, and retrieval. Semantic Web Rules express domain-specific rules and logic for reasoning. When videos are classified and tagged by the VBDML then the respective IR are persistent to VBDCL and also mapped to the Video Ontology while using the Feature0nto Mapper. Finally, SPARQL based semantic rich queries are allowed for knowledge graph, complex event reasoning, analysis, and retrieval.

\section{Research Issues, Opportunities, and Future Directions}

Intelligent video big data analytics in the cloud opens new research avenues, challenges, and opportunities. This section provides in-depth detail about such research challenges (summarized in Table 1).

IVA on Video Big data: Big data analytics engines are the general-purpose engine and are not mainly designed for big video analytics. Consequently, video big data analytics is challenging over such engines and demand optimization. Almost all the engines are inherently lacking the support of elementary video data structures and processing operations. Further, such engines are also not optimized, especially for iterative IVA and dependency among processes. Furthermore, the focus of the existing research on IVA are velocity, volume, velocity, but the veracity and value have been overlooked. One promising direction in addressing video big data veracity is to research methods and techniques capable 
Table 1. Video Big Data Analytics Open Research Issues and Challenges in the Cloud.

\begin{tabular}{|c|c|c|c|}
\hline Component & Aspect & Layer & Open Research Issues \\
\hline \multirow[t]{7}{*}{ Video Big Data } & Volume & VBDCL & Orchestration and Optimization of IVA Pipeline \\
\hline & & VBDCL, VBDPL, VBDML & Big dimensionality reduction, and indexing \\
\hline & & & Cleaning and compressing video big data \\
\hline & Velocity & VBDCL, VBDPL, VBDML & Real-time video streams and online learning \\
\hline & Variety & & $\begin{array}{l}\text { Big dimensionality reduction } \\
\text { Data modality for single IVA goal }\end{array}$ \\
\hline & Veracity & & $\begin{array}{l}\text { Vide big data veracity assessment } \\
\text { Learning with unreliable data }\end{array}$ \\
\hline & Value & & Understandable IVA for decision support \\
\hline & & & Semantic concepts extraction in distributed environment \\
\hline \multirow[t]{7}{*}{ User } & $\begin{array}{l}\text { Developer / } \\
\text { Researcher }\end{array}$ & WSL & $\begin{array}{l}\text { Declarative IVA } \\
\text { IVA and distributed computing technologies abstraction. } \\
\text { Comprehensive evaluation measures for IVA in the cloud environment } \\
\text { Visualizing video big data }\end{array}$ \\
\hline & & VBDCL, WSL & IVA algorithm, model, and services statistics maintenance and ranking \\
\hline & & & Effective price scheme for IVA algorithm deployment, and subscription \\
\hline & & WSL, VBDCL & Model management and algorithm selection \\
\hline & Consumer & WSL & $\begin{array}{l}\text { IVA services utilization } \\
\text { Improving consumer experience based on feedback }\end{array}$ \\
\hline & & $\begin{array}{l}\text { VBDCL, VBDPL, } \\
\text { VBDML, VKCL }\end{array}$ & Effective price scheme for multiple IVA service subscription \\
\hline & Security and Privacy & & Privacy preserving distributed IVA, security, and trust \\
\hline \multirow[t]{2}{*}{ Cloud System } & Analytics engine & VBDPL, VBDML & $\begin{array}{l}\text { IVA on video big data (general big data middleware for IVA) } \\
\text { Parameter Server optimization }\end{array}$ \\
\hline & Infrastructure & & IVA on video big data (general big data middleware for IVA) \\
\hline
\end{tabular}

of accessing the credibility of video data sources so that untrustworthy video data can be filtered. Another way is to come up with novel ML models that can make inferences with insufficient video data. Likewise, users' assistance is required to comprehend IVA results and the reason behind the decision to realize the value of video big data in decision support. Thus, understandable IVA can be a significant future research area.

IVA and Human-machine coordination: IVA on video big data grants a remarkable opportunity for learning with human-machine coordination for numerous reasons. IVA on video big data in cloud demands researchers and practitioners mastering both IVA and distributed computing technologies. Bridging both the worlds for most analysts is challenging. Especially in an educational environment, where the researcher focuses more on the understanding, configuration, and tons of parameters rather than innovation and research contribution. Thus there is a growing need to design such L-CVAS that provide high-level abstractions to hide the underlying complexity. IVA service to become commercially worthwhile and to achieve pervasive recognition, consumer lacking technical IVA knowledge. The consumers should be able to configure, subscribe, and maintain IVA services with comfort. In traditional IVA, consumers are usually passive. Further, research is required to build more interactive IVA services that assist consumers in gaining insight into video big data.

Orchestration and Optimization of IVA Pipeline: The real-time and batch workflow are deeply dependent on the messaging middleware and distributed processing engines. The dynamic (R/B)IVA service creation and multisubscription environment demand the optimization and orchestration of the IVA service pipeline 3] while guarantees opportunities for further research. In the map-reduce infrastructure, a slowdown predictor can be utilized to improve the agility and timeliness of scheduling decisions. Spark and Flink can accumulate 
a sequence of algorithms into a single pipeline but need research to examine its behavior in dynamic service creation and subscription environment. Further, concepts from the field of query and queuing optimization can be utilized while considering messaging middleware and distributed processing engines with the aim of orchestrating and optimization of IVA service Pipeline.

IVA and Big Dimensionality: The VSDS multi-modality can produce diverse types of data streams. Similarly, algorithms generate varied sorts of multi-dimensional features. The high-dimensionality factor poses many intrinsic challenges for data stream acquisition, transmission, learner, pattern recognition problems, indexing, and retrieval. In literature, it has been referred to as a "Big Dimensionality" challenge 43. VSDS variety leads to key challenges is how to acquire and process the heterogeneous data in an effective way. Most existing IVA approaches can consider a specific input, but in many cases, for a single IVA goal, different kinds, and formats can be considered. With growing features dimensionality, current algorithms quickly become computationally inflexible and, therefore, inapplicable in many real-time applications [16. Dimension reduction approaches are still a hot research topic because of data diversity, increasing volume, and complexity. Effect-learning algorithms for first-order optimization, online learning, and paralleling computing will be more preferred.

Model management: An algorithm might hold a list of parameters. The model selection process encompasses feature engineering, IVA algorithm selection, and hyperparameter tuning. Feature engineering is a strenuous activity and is influenced by many key factors, e.g., domain-specific regulations, time, accuracy, video data, and IVA properties, which resultantly slow and hinder exploration. IVA algorithm selection is the process of choosing a model that fixes the hypothesis space of prediction function explored for a given application [15. This process of IVA algorithm selection is reliant on technical and non-technical aspects, which enforce the IVA developer to try manifold techniques at the cost of time and cloud resources. Hyperparameter is vital as they govern the tradeoffs between accuracy and performance. IVA analysts usually do ad-hoc manual tuning by iteratively choosing a set of values or using heuristics such as grid search [15]. From IVA analysts' perspective, model selection is an expensive job in terms of time and resources that bringing down the video analytics lifecycle. Model selection is an iterative and investigative process that generally creates an endless space, and it is challenging for IVA analysts to know a priori which combination will produce acceptable accuracy/insights. In this direction, theoretical design trade-offs are presented by Arun et al. 25, but further research is required that how to shape a unified framework that acts as a foundation for a novel class of IVA analytics while building the procedure of model selection easier and quicker.

Statistics maintenance and ranking: A user can develop and deploy an IVA algorithm, model, or service that can be either extended, utilized, or subscribed by other users. The community members run such architecture, and rapidly, the number of IVA services can be reached to tons of domain-dependent or independent IVA services. This scenario develops a complex situation for the 
users, i.e., which IVA service (when sharing the parallel functionalities) in a specific situation, especially during service discover. Against each IVA service, there is a list of Quality of Service (QoS) parameters. Some of these QoS parameters (not limited to) are user trust, satisfaction, domain relevance, security, usability, availability, reliability, documentation, latency, response time, resource utilization, accuracy, and precision. Such types of IVA services against the QoS parameters lead to the 0-1 knapsack issue. In this direction, one possible solution is utilizing multi-criteria decision-making approaches. It gives further opportunities to the research community to investigate how to rank and recommend IVA algorithms, models, and services.

IVAaaS and Cost Model: The proposed L-CVAS is supposed to provide IVA-Algorithm-as-a-Service (IVAAaaS) and IVA-as-a-Service (IVAaaS) in the cloud while adopting the Customer-to-Customer (C2C) business model. Unfortunately, current Software-as-a-Service (SaaS) cost models might not be applicable because of the involvement of diverse types of parameters that drastically affect the cost model. Such parameters are, business model (Business-to-Business (B2B), Business-to-Customer (B2C), and C2C), unite of video, user type (developer, researcher, and consumers), services (IVAAaaS and IVAaaS), service subscription (algorithm, IVA service, single, multiple, dependent or independent), cloud resource utilization, user satisfaction, QoS, location, service subscription duration, and cost model fairness. The addition of further parameters is subject to discussion, but the listed are the basic that govern L-CVAS cost matrix. Additionally, the cost model demands further research and investigations to develop an effective price scheme for IVA services while considering the stated parameters.

Video big data management: Despite video big data pose high value, but its management, indexing, retrieval, and mining are challenging because of its volume, velocity, and unstructuredness 21. In the context of video big data management, the main issue is the extraction of semantic concepts from primitive features. A general domain-independent framework is required that can extract semantic features, analyze and model the multiple semantics from the videos by using the primitive features. Further, semantic event detection is still an open research issue because of the semantic gap and the difficulty of modeling temporal and multi-modality features of video streams. The temporal information is significant in the video big data mining mainly, in pattern recognition. Limited research is available on content-based video retrieval while exploiting distributed computing. Further study is required to consider different types of features ranging from local to global spatiotemporal features utilizing and optimizing deep learning and distributed computing engines. Semantic-based approaches have been utilized for video retrieval because of the semantic gap between the lowlevel features and high-level human-understandable concepts [2]. Ontology adds extra concepts that can improve the retrieval results and lead to unexpected deterioration of search results. In this context, a hybrid approach can be fruitful and need to design different query planes that can fulfill diverse queries in complex situations. 
Privacy, Security and Trust: Video big data, acquisition, storage, and subscriptions to shared IVA in the cloud become mandatory, which leads to privacy concerns. For the success of such platforms, privacy, security, and trust are always central. In literature, the word 'trust' is commonly used as a general term for 'security' and 'privacy'. Trust is a social phenomenon where the user has expectations from the IVA service provider and willing to take action (subscription) on the belief based on evidence that the expected behavior occurs [22]. In the cloud environment, security and privacy are playing an active role in the trust-building. To ensure security, the L-CVAS should offer different levels of privacy control. The privacy and security phenomena are valid across VSDS, storage security, multi-level access controls, and privacy-aware IVA and analysis.

\section{Conclusion}

In the recent past, the number of public surveillance cameras has increased significantly, and an enormous amount of visual data is produced at an alarming rate. Such large-scale video data pose the characteristics of big data. Video big data offer opportunities to the video surveillance industry and permits them to gain insights in almost real-time. The deployment of big data technologies such as Hadoop, Spark, etc., in the cloud under aaS paradigm to acquire, persist, process and analyze a large amount of data has been in service from last few years. This approach has changed the context of information technology and has turned the on-demand service model's assurances into reality. This paper presents a comprehensive layered architecture for intelligent video big data analytics in the cloud under the aaS. Furthermore, research issues, opportunity, and challenges being raised by the uniqueness of the proposed CVAS, and the triangular relation among video big data analytics, distributed computing technologies, and cloud has been reported. 
A. Alam et al. 


\title{
List of abbreviations
}

\author{
aaS as-a-Service. \\ B2B Business-to-Business. \\ B2C Business-to-Customer. \\ BIVA Batch IVA. \\ C2C Customer-to-Customer. \\ CNN Convolutional Neural Network. \\ CVAS Cloud-based Video Analytics System. \\ DAG Directed Acyclic Graph. \\ DBDS Distributed Big Datastore. \\ DFS Distributed File System. \\ DMBM Distributed Message Broker Manager. \\ DPDS Distributed Persistent Data Store. \\ HDFS Hadoop File System. \\ IoT Internet of Things. \\ IR Intermediate Results. \\ IRM Intermediate Results Manager. \\ ISDDS Immediate Structured Distributed Data Store. \\ IVA Intelligent Video Analytics. \\ IVAAaaS IVA-Algorithm-as-a-Service. \\ IVAaaS IVA-as-a-Service. \\ KCL Knowledge Curation Layer. \\ L-CVAS Lambda CVAS. \\ LVSM Lifelong Video Stream Monitor. \\ ML Machine Learning. \\ PCA Principal Component Analysis. \\ QoS Quality of Service. \\ RIVA Real-time IVA. \\ RVSAS Real-time Video Stream Acquisition and Syn- \\ chronization. \\ SaaS Software-as-a-Service. \\ SVM Support Vector Machine. \\ UPDDS Unstructured Persistent Distributed Data \\ Store. \\ VBDCL Video Big Data Curation Layer. \\ VBDML Video Big Data Mining Layer. \\ VBDPL Video Big Data Processing Layer. \\ VSC Video Stream Consumer. \\ VSDS Video Stream Data Source. \\ VSP Video Stream Producer. \\ WSL Web Service Layer.
}




\section{References}

1. Abadi, M., Barham, P., Chen, J., Chen, Z., Davis, A., Dean, J., Devin, M., Ghemawat, S., Irving, G., Isard, M., et al.: Tensorflow: A system for large-scale machine learning. In: OSDI. vol. 16, pp. 265-283 (2016)

2. Alam, A., Khan, M.N., Khan, J., Lee, Y.K.: Intellibvr-intelligent large-scale video retrieval for objects and events utilizing distributed deep-learning and semantic approaches. In: 2020 IEEE BigComp. pp. 28-35. IEEE (2020)

3. Alam, A., Lee, Y.K.: Tornado: Intermediate results orchestration based serviceoriented data curation framework for intelligent video big data analytics in the cloud. Sensors 20(12), 3581 (2020)

4. Amazon, E.: Amazon web services. Available in: http://aws. amazon. com/es/ec2/(November 2012) (2015)

5. Borisyuk, F., Gordo, A., Sivakumar, V.: Rosetta: Large scale system for text detection and recognition in images. In: Proceedings of the 24th ACM SIGKDD International Conference on Knowledge Discovery \& Data Mining. pp. 71-79. ACM (2018)

6. Carbone, P., Katsifodimos, A., Ewen, S., Markl, V., Haridi, S., Tzoumas, K.: Apache flink: Stream and batch processing in a single engine. Bulletin of the IEEE Computer Society Technical Committee on Data Engineering 36(4) (2015)

7. Chollet, F., et al.: Keras (2015)

8. Corporation, I.D.: The growth in connected iot devices (2019), https://www.idc.com/getdoc.jsp?containerId=prUS45213219, accessed: 2019-12-07

9. Dai, J., Wang, Y., Qiu, X., Ding, D., Zhang, Y., Wang, Y., Jia, X., Zhang, C., Wan, Y., Li, Z., et al.: Bigdl: A distributed deep learning framework for big data. arXiv preprint arXiv:1804.05839 (2018)

10. Dean, J., Corrado, G., Monga, R., Chen, K., Devin, M., Mao, M., Ranzato, M., Senior, A., Tucker, P., Yang, K., et al.: Large scale distributed deep networks. In: Advances in neural information processing systems. pp. 1223-1231 (2012)

11. Dean, J., Ghemawat, S.: Mapreduce: a flexible data processing tool. Communications of the ACM 53(1), 72-77 (2010)

12. Deshpande, A., Kumar, M.: Artificial Intelligence for Big Data: Complete Guide to Automating Big Data Solutions Using Artificial Intelligence Techniques. Packt Publishing Ltd (2018)

13. Dijcks, J.P.: Oracle: Big data for the enterprise. Oracle white paper p. 16 (2012)

14. Facebook.Com: Facebook (2020), https://www.facebook.com accessed: 2019-1220

15. Friedman, J., Hastie, T., Tibshirani, R.: The elements of statistical learning, vol. 1. Springer series in statistics New York (2001)

16. Gao, L., Song, J., Liu, X., Shao, J., Liu, J., Shao, J.: Learning in high-dimensional multimedia data: the state of the art. Multimedia Systems 23(3), 303-313 (2017)

17. He, K., Zhang, X., Ren, S., Sun, J.: Deep residual learning for image recognition. In: Proceedings of the IEEE conference on computer vision and pattern recognition. pp. $770-778$ (2016)

18. Hestness, J., Narang, S., Ardalani, N., Diamos, G., Jun, H., Kianinejad, H., Patwary, M., Ali, M., Yang, Y., Zhou, Y.: Deep learning scaling is predictable, empirically. arXiv preprint arXiv:1712.00409 (2017)

19. Huang, T.: Surveillance video: The biggest big data. Computing Now $\mathbf{7}(2), 82-91$ (2014) 
20. Ketkar, N.: Introduction to pytorch. In: Deep learning with python, pp. 195-208. Springer (2017)

21. Khan, M.N., Alam, A., Lee, Y.K.: Falkon: Large-scale content-based video retrieval utilizing deep-features and distributed in-memory computing. In: 2020 IEEE International Conference on Big Data and Smart Computing (BigComp). pp. 36-43. IEEE (2020)

22. Khusro, S., Alam, A., Khalid, S.: Social question and answer sites: the story so far. Program 51(2), 170-192 (2017)

23. Kreps, J., Narkhede, N., Rao, J., et al.: Kafka: A distributed messaging system for log processing. In: Proceedings of the NetDB. pp. 1-7 (2011)

24. Krizhevsky, A., Sutskever, I., Hinton, G.E.: Imagenet classification with deep convolutional neural networks. In: Advances in neural information processing systems. pp. 1097-1105 (2012)

25. Kumar, A., McCann, R., Naughton, J., Patel, J.M.: Model selection management systems: The next frontier of advanced analytics. ACM SIGMOD Record 44(4), $17-22(2016)$

26. LeCun, Y., Bengio, Y., Hinton, G.: Deep learning. nature 521(7553), 436 (2015)

27. Marz, N., Warren, J.: Big Data: Principles and best practices of scalable realtime data systems. New York; Manning Publications Co. (2015)

28. Mayer, R., Jacobsen, H.A.: Scalable deep learning on distributed infrastructures: Challenges, techniques and tools. arXiv preprint arXiv:1903.11314 (2019)

29. Meng, X., Bradley, J., Yavuz, B., Sparks, E., Venkataraman, S., Liu, D., Freeman, J., Tsai, D., Amde, M., Owen, S., et al.: Mllib: Machine learning in apache spark. The Journal of Machine Learning Research 17(1), 1235-1241 (2016)

30. Olatunji, I.E., Cheng, C.H.: Dynamic threshold for resource tracking in observed scenes. In: 2018 9th International Conference on Information, Intelligence, Systems and Applications (IISA). pp. 1-6. IEEE (2018)

31. Owen, S., Owen, S.: Mahout in action (2012)

32. Pouyanfar, S., Yang, Y., Chen, S.C., Shyu, M.L., Iyengar, S.: Multimedia big data analytics: A survey. ACM Computing Surveys (CSUR) 51(1), 10 (2018)

33. Simonyan, K., Zisserman, A.: Very deep convolutional networks for large-scale image recognition. arXiv preprint arXiv:1409.1556 (2014)

34. Szegedy, C., Liu, W., Jia, Y., Sermanet, P., Reed, S., Anguelov, D., Erhan, D., Vanhoucke, V., Rabinovich, A.: Going deeper with convolutions. In: Computer Vision and Pattern Recognition (CVPR) (2015), http://arxiv.org/abs/1409.4842

35. Team, D., et al.: Deeplearning4j: Open-source distributed deep learning for the jvm. Apache Software Foundation License 2 (2016)

36. Uddin, M.A., Lee, Y.K.: Feature fusion of deep spatial features and handcrafted spatiotemporal features for human action recognition. Sensors 19(7), 1599 (2019)

37. Vora, M.N.: Hadoop-hbase for large-scale data. In: Computer science and network technology (ICCSNT), 2011 international conference on. vol. 1, pp. 601-605. IEEE (2011)

38. Xie, L., Sundaram, H., Campbell, M.: Event mining in multimedia streams. Proceedings of the IEEE 96(4), 623-647 (2008)

39. youtube.com: Youtube statistics (2019), https://www.youtube.com/about/press/ accessed: 2019-12-20

40. Yue-Hei Ng, J., Hausknecht, M., Vijayanarasimhan, S., Vinyals, O., Monga, R., Toderici, G.: Beyond short snippets: Deep networks for video classification. In: Proceedings of the IEEE conference on computer vision and pattern recognition. pp. 4694-4702 (2015) 
41. Zaharia, M., Das, T., Li, H., Hunter, T., Shenker, S., Stoica, I.: Discretized streams: Fault-tolerant streaming computation at scale. In: Proceedings of the twenty-fourth ACM symposium on operating systems principles. pp. 423-438. ACM (2013)

42. Zaharia, M., Xin, R.S., Wendell, P., Das, T., Armbrust, M., Dave, A., Meng, X., Rosen, J., Venkataraman, S., Franklin, M.J., et al.: Apache spark: a unified engine for big data processing. Communications of the ACM 59(11), 56-65 (2016)

43. Zhai, Y., Ong, Y.S., Tsang, I.W.: The emerging" big dimensionality" (2014)

44. Zhang, H., Zheng, Z., Xu, S., Dai, W., Ho, Q., Liang, X., Hu, Z., Wei, J., Xie, P., Xing, E.P.: Poseidon: An efficient communication architecture for distributed deep learning on $\{$ GPU\} clusters. In: 2017 \{USENIX\} Annual Technical Conference ( $\{$ USENIX $\}\{$ ATC $\} 17)$. pp. 181-193 (2017) 